\title{
Association between unintentional splenic radiation and lymphopenia and high neutrophil/lymphocyte ratio after radiotherapy in patients with esophageal cancer
}

\author{
Masakuni Sakaguchi, Toshiya Maebayashi, Takuya Aizawa, Naoya Ishibashi, Masahiro Okada \\ Department of Radiology, Nihon University School of Medicine, Itabashi-ku, Tokyo, Japan \\ Contributions: (I) Conception and design: M Sakaguchi; (II) Administrative support: T Maebayashi, M Okada; (III) Provision of study materials \\ or patients: M Sakaguchi, T Maebayashi, T Aizawa, N Ishibashi; (IV) Collection and assembly of data: M Sakaguchi; (V) Data analysis and \\ interpretation: M Sakaguchi; (VI) Manuscript writing: All authors; (VII) Final approval of manuscript: All authors. \\ Correspondence to: Masakuni Sakaguchi. Department of Radiology, Nihon University School of Medicine, 30-1, Oyaguchi Kami-cho, Itabashi-ku, \\ Tokyo 173-8610, Japan. Email: sakaguchi.masakuni@nihon-u.ac.jp.
}

\begin{abstract}
Background: Unintentional irradiation of the spleen may reduce absolute lymphocyte count (ALC), which can affect tumor immunity. Therefore, in the present study, we evaluated spleen dose-volume parameters associated with ALC and neutrophil/lymphocyte ratio (NLR) in patients with esophageal cancer.

Methods: This retrospective study evaluated patients who were diagnosed with stage I-IV esophageal cancer and who received radiotherapy at Nihon University Itabashi Hospital between January 2015 and March 2020. Spleen V5, V10, V20, and V30 and mean spleen dose, which are dose-volume histogram parameters correlated with ALC and NLR, were analyzed.

Results: In total, 89 esophageal cancer patients with a median age of 72 years (range, 51-92 years) were included in this research. Results showed that spleen V5, V10, and V20 and mean splenic dose were significantly correlated with a low ALC. Meanwhile, a significant association was observed between spleen V5 and V10 and a high NLR $(\mathrm{P}<0.05)$. In the linear regression analysis, spleen V5 and V10 were remarkably associated with a low ALC $(\mathrm{P}=0.006$ and 0.008$)$. Further, a correlation was noted between spleen V5 and a high NLR ( $\mathrm{P}=0.019)$. Spleen V5 and V10 were remarkably associated with greater than grade 3 lymphopenia $(\mathrm{P}=0.024$ and $\mathrm{P}=0.031)$.

Conclusions: A correlation was observed between the irradiated volume of the spleen and ALC and NLR in patients with esophageal cancer. Furthermore, the spleen should be regarded as a high-risk organ, and the use of techniques in reducing spleen V5, V10 for the preservation of tumor immunity may be beneficial.
\end{abstract}

Keywords: Absolute lymphocyte count (ALC); esophageal cancer; neutrophil/lymphocyte ratio (NLR); radiotherapy

Submitted Aug 28, 2021. Accepted for publication Nov 12, 2021.

doi: $10.21037 /$ tcr-21-1765

View this article at: https://dx.doi.org/10.21037/tcr-21-1765

\section{Introduction}

Chemoradiotherapy is widely used for unresectable esophageal cancer, and its treatment outcomes have significantly improved $(1,2)$. However, the incidence of acute and late adverse events may increase due to the use of combined chemotherapy and the widespread irradiation field (3). Since radiotherapy (RT) is applied for the management of esophageal cancer, an X-ray beam is exposed via the anterior and posterior opposed portals, and an oblique opposed portal for the primary lesion and lymph node metastasis is often utilized. The late side effects of RT include pulmonary disorders and pericardial effusion, and caution is observed for these high-risk organs when establishing treatment plans. However, oblique beams 
often unintentionally pass through the spleen, which is the most important structure of the lymphoid tissues and is the organ where peripheral lymphocytes that circulate in the body pass constantly (4). Irradiation of the spleen may be directly associated with lymphopenia in the peripheral blood because lymphocytes are highly radiosensitive. Lymphocytes, which play a role in anti-tumor immunity, can specifically recognize, and attack tumor cells and release several cytokines to activate the immune system of a host (5). The use of decreased absolute lymphocyte count (ALC) and elevated neutrophil/lymphocyte ratio (NLR) before treatment as predictive or prognostic factors for various cancers, including esophageal cancer, has attracted significant attention (6-10). However, only few reports existed on the association between spleen irradiation and ALC and NLR in patients with esophageal cancer. Furthermore, previous studies have only assessed ALC after treatment (11), and no research has evaluated NLR changes before and after RT or concurrent chemoradiotherapy (CCRT). Before treatment, patients with advanced-stage disease and poor prognosis may present with a low ALC and a high NLR (8). When assessing the effect of RT or CCRT on ALC or NLR, changes in these parameters before and after treatment must be evaluated. Thus, the present study first evaluated the effect of spleen dose-volume parameters on ALC and change of NLR in patients with esophageal cancer.

We present the following article in accordance with the STROBE reporting checklist. (Available at https://dx.doi. org/10.21037/tcr-21-1765).

\section{Methods}

\section{Characteristics of the patients}

Esophageal cancer patients who received RT at Nihon University Itabashi Hospital between January 2015 and March 2020 and who have available medical records were retrospectively assessed. Because the treatment planning system in our hospital was replaced in 2014, we only had access to treatment plans from 2015 . Patients with concurrent hematologic, autoimmune, or systemic inflammatory diseases who previously received chemotherapy for other types of cancer were excluded. All participants were histologically diagnosed with esophageal cancer, and their lesions were staged according to the seventh edition of the tumor, node, metastasis (TNM) classification system (12). All patients underwent computed tomography (CT) scan for the evaluation of the primary site and lymph node and distant metastases. Patients who were enrolled more recently underwent positron emission tomography.

\section{Treatment methods}

The treatment regimen basically included 5-fluorouracil (5-FU) combined with cisplatin (CDDP) with adequate hydration and antiemetic coverage (FP regimen). Two cycles of chemotherapy were administered every 4 weeks during RT. Some patients with advanced-stage received combined CDDP and 5-FU and docetaxel, and irinotecan and CDDP were used for small cell carcinoma. We selected RT alone because of advanced age and renal impairment. A $10-M V X$-ray beam was exposed via the anterior and posterior opposed portals, and an oblique opposed portal was commonly used. Each beam was created by adjusting the planning target volume with a margin along the path of the beam. Intensity-modulated RT was not used because our institution had no established policy for the use of this treatment modality in patients with esophageal cancer. RT was delivered 5 days a week using a single daily fraction of 2 Gy. None of the patients received hyperfractionated RT. The total doses used were 40 Gy for preoperative CCRT and 60 Gy for radical CCRT. Since radiation therapy was administered at a dose of $>40 \mathrm{~Gy}$, all patients underwent a CT scan, and re-planned RT was conducted in only oblique opposed fields to exclude the spinal cord.

\section{Treatment planning and spleen dosimetry}

The spleen was contoured in each patient, and its corresponding dosimetry was calculated by a treatment machine [Monaco 5.11 .02 (Elekta)]. The total dose to the spleen was determined by combining the first and second plans ( $>40 \mathrm{~Gy}$ ) in patients who received a dose of $>40 \mathrm{~Gy}$. Five specific dose-volume histogram parameters (i.e., V5, V10, V20, V30, and mean spleen dose) were selected for the correlational analysis. For example, V5 indicated a volume of 5 Gy.

\section{Blood collection}

Blood samples were collected from all patients to evaluate the lymphocyte and neutrophil counts before and after treatment. We referred to blood samples collected 
Table 1 Baseline clinical characteristics of patients

\begin{tabular}{|c|c|}
\hline Characteristics & Values \\
\hline Patients & 89 \\
\hline Age (years), median (range) & $72(51-92)$ \\
\hline \multicolumn{2}{|l|}{ Gender } \\
\hline Male & $71(80 \%)$ \\
\hline Female & $18(20 \%)$ \\
\hline \multicolumn{2}{|l|}{ PS } \\
\hline 0 & $3(3 \%)$ \\
\hline 1 & $71(80 \%)$ \\
\hline 2 & $15(17 \%)$ \\
\hline \multicolumn{2}{|l|}{ Location } \\
\hline Upper & $6(6 \%)$ \\
\hline Middle & $49(55 \%)$ \\
\hline Lower & $34(39 \%)$ \\
\hline \multicolumn{2}{|l|}{ Pathology } \\
\hline Squamous & $86(97 \%)$ \\
\hline Adeno squamous & $1(1 \%)$ \\
\hline Adeno & $1(1 \%)$ \\
\hline Small & $1(1 \%)$ \\
\hline \multicolumn{2}{|l|}{ Stage } \\
\hline I & $11(12 \%)$ \\
\hline II & $13(15 \%)$ \\
\hline III & $58(65 \%)$ \\
\hline IV & $7(8 \%)$ \\
\hline \multicolumn{2}{|l|}{ Radiation dose } \\
\hline 40 & $54(61 \%)$ \\
\hline 50 & $10(11 \%)$ \\
\hline 60 & $21(24 \%)$ \\
\hline Other & $4(4 \%)$ \\
\hline \multicolumn{2}{|l|}{ Treatment } \\
\hline RT alone & $9(10 \%)$ \\
\hline CCRT & $80(90 \%)$ \\
\hline $\mathrm{CDDP}+5-\mathrm{Fu}$ & $78(98 \%)$ \\
\hline DCF & $1(1 \%)$ \\
\hline CPT11 + CDDP & $1(1 \%)$ \\
\hline
\end{tabular}

PS, performance status; stage, TNM classification 7th edition; $\mathrm{RT}$, radiotherapy; CCRT, concurrent chemoradiation therapy; CDDP, cisplatin; 5-FU, 5-fluorouracil; DCF, docetaxel, cisplatin, 5-fluorouracil; CPT-11, irinotecan. immediately before the start of treatment (on the day or within a few before). If blood collection before treatment was not performed immediately, blood collection at the first visit (within 1 month) was used as a reference. The ALC and neutrophil count were based on blood sampling that was conducted immediately after treatment completion. When the period from the end of treatment to blood collection was $\geq 1$ week, the result obtained immediately before the end of treatment was used as a reference. According to the Common Terminology Criteria for Adverse Events version 4.0, more than G3 lymphopenia was defined as $<500 / \mu \mathrm{L}$. NLR was defined as neutrophil count divided by lymphocyte count. Increase ratio of NLR was calculated by dividing the NLR after RT by the NLR before RT.

\section{Statistical analysis}

Using the Mann-Whitney $U$ test, the median ALC and NLR before and after RT were compared. The Spearman correlation coefficients were used to evaluate the associations between the spleen dose-volume parameters and the ALC and NLR. A higher NLR was obtained by dividing the NLR after RT by the NLR before RT. In the univariate linear regression analyses, the ALC, NLR, and dose-volume parameters were considered as continuous variables. Overall survival (OS) was defined as the period from the last day of RT to the time of death regardless of the cause. Using the Kaplan-Meier method, OS was evaluated. A P value of $<0.05$ was considered as statistically significant.

\section{Ethical statement}

The study was conducted in accordance with the Declaration of Helsinki (as revised in 2013). This study was approved by our institutional review board of Nihon University Itabashi Hospital (Ref No. RK-180213-9). All patients provided consent for their data to be used and additional consent for this retrospective analysis was waived.

\section{Results}

\section{Patient and treatment characteristics}

Table 1 contains the baseline patient and treatment characteristics. In total, 89 patients who fulfilled the criteria were included in the study. The median age of the participants was 72 years (range, 51-92 years). The proportion of patients with stage III cancer was the 
highest, followed by those with stage II cancer. Squamous cell carcinoma was the most common histopathological diagnosis, followed by adenocarcinoma, adenosquamous cell carcinoma, and small cell carcinoma. FP-based CCRT was the most frequent treatment, followed by RT monotherapy, because of poor general condition and/or renal dysfunction.

\section{Blood examination}

The median ALCs before and after treatment were $1,562 / \mu \mathrm{L}$ (range, $470-3,213 / \mu \mathrm{L}$ ) and $333 / \mu \mathrm{L}$ (range, $89-1,328 / \mu \mathrm{L}$ ), respectively. Of 89 patients, $76(85.4 \%)$ presented with greater than grade 3 lymphopenia immediately after treatment. The median NLRs before and after treatment were 2.83 (range, 0.80-11.16) and 7.84 (range, 1.7189.51 ), respectively. The Mann-Whitney $U$ test showed a significant difference between the median ALC and NLR before and after RT $(\mathrm{P}<0.0001)$.

\section{Dose-volume histogram analysis}

Based on the dose-volume histogram analysis of the spleen derived using the CT scan datasets, the median V5, V10, V20, and V30 were $35.0 \mathrm{~mL}$ (range, 0-119.50 mL), $21.5 \mathrm{~mL}$ (range, 0-115.1 mL), $3.88 \mathrm{~mL}$ (range, 0-87.0 mL), and $0.54 \mathrm{~mL}$ (range, $0-71.9 \mathrm{~mL}$ ), respectively. The mean splenic dose was 6.4 Gy (range, 0.01-32.8 Gy).

\section{Correlations between splenic dose parameters and ALC and NLR}

The Spearman correlation analysis revealed that V5, V10, V20, and mean splenic dose were significantly correlated with decreased ALC (P=0.0005, 0.0002, 0.0223, and 0.0009, respectively; Figure 1). This association subsequently decreased at higher doses delivered to the spleen (V30). V5 and V10 were significantly correlated with a high NLR ( $\mathrm{P}=0.004,0.008$, respectively; Figure 2), and the no association at V20, V30, mean spleen dose.

\section{Predictors of splenic dose parameters}

In the linear regression analysis, spleen V5 and V10 were found to be significantly associated with a low ALC $(\mathrm{P}=0.006$ and 0.008$)$. Moreover, spleen V5 was associated with a high NLR $(\mathrm{P}=0.019)$ (Table 2). A logistic regression analysis was conducted to determine whether V5 and V10 were significantly associated with greater than grade 3 lymphopenia (Table 3).

\section{Relationship between lymphopenia (grades 1 and 2 vs. 3 and 4) and $O S$}

The median follow-up period from the last day of RT was 12 months (range, 2-59 months). No statistically significant difference was found in OS between patients with grades 1 and 2 cancer and those with grades 3 and 4 cancer $(\mathrm{P}=0.2406)$.

\section{Discussion}

The spleen is the largest lymphoid tissue in the body. In particular, the white pulp activates the immune response when antigens and antibodies are present in the blood $(13,14)$. An unintended increase in spleen dose can have a significant impact on ALC and tumor immunity. Our study demonstrated that spleen dose was shown to be associated with decrease ALC and increase ratio of NLR after treatment in patients with esophageal cancer.

A previous study has shown a significant correlation between high spleen irradiation doses and low ALC after $\mathrm{RT}$ in patients with hepatocellular carcinoma and pancreatic cancer $(10,15)$. Our results are similar to those of previous reports showing that patients with esophageal cancer have a low ALC, which is attributed to the splenic dose of RT. Of note, in terms of V5, V10, V20, V30, and mean splenic dose, the delivery of low doses (i.e., V5) to the spleen was more strongly associated with a low ALC. Based on previous research that used a micronucleus assay for detecting $\mathrm{X}$-rayinduced chromosome damage in lymphocytes, damage to lymphocytes was found to occur at low doses $(16,17)$. Other studies showed that the lethal doses required to reduce the surviving fraction of lymphocytes by $50 \%$ and $90 \%$ are approximately 2 and 3 Gy, respectively $(18,19)$. Our findings are consistent with those of previous reports. Therefore, since lymphocytes are affected, low doses (i.e., V5), rather than high doses, delivered to the spleen are an extremely important predictor of lymphopenia.

Recent studies have shown that inflammation-related prognostic factors, including NLR, may be used as a parameter of systemic inflammation in various cancers including esophageal cancer $(8,20,21)$. Inflammatory pathways play a key role in tumorigenesis, tumor-related angiogenesis, and tumor invasion/metastasis $(22,23)$. A high 

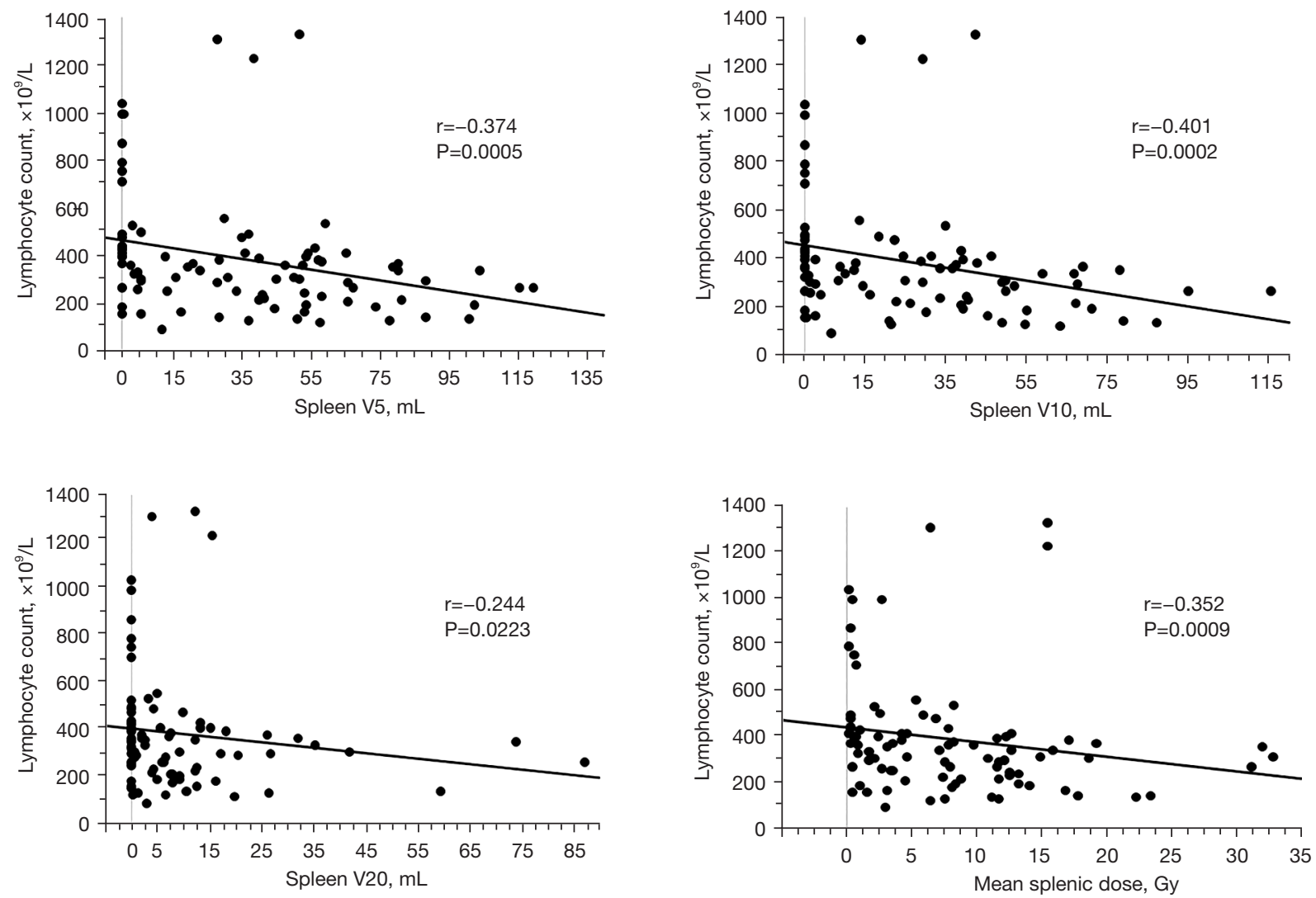

Figure 1 Correlations between spleen dose volume parameters and ALC during radiotherapy in patients with esophageal cancer. ALC, absolute lymphocyte count.
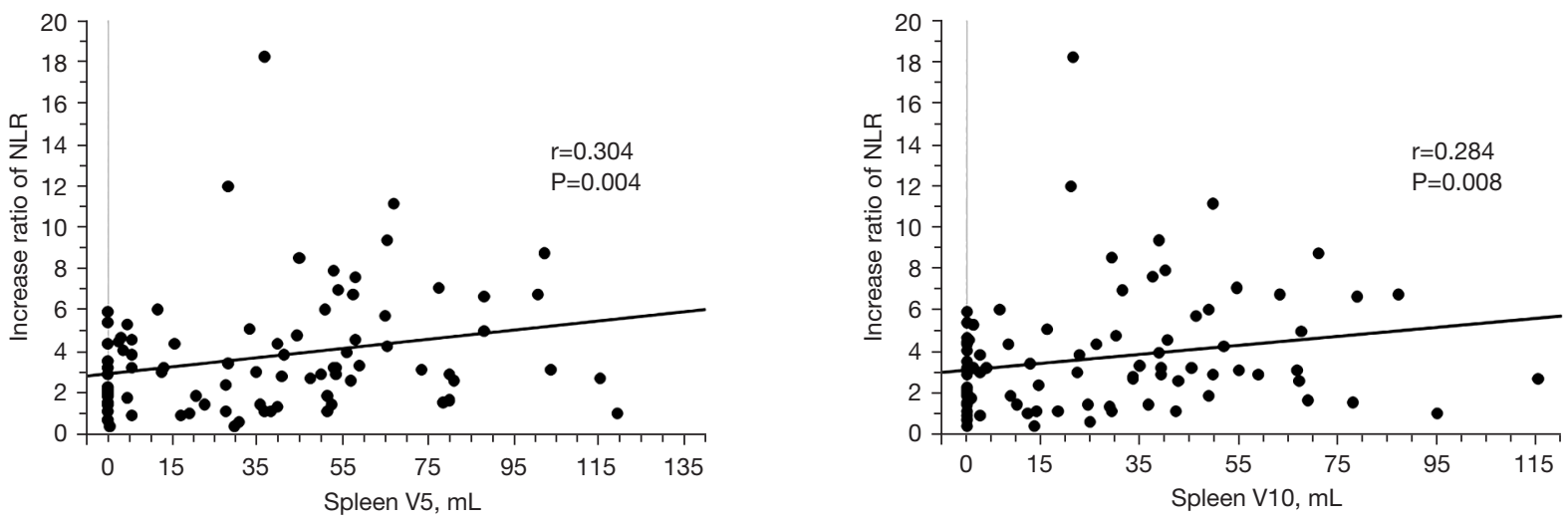

Figure 2 Correlations between spleen dose volume parameters and NLR during radiotherapy in patients with esophageal cancer. NLR, neutrophil/lymphocyte ratio.

NLR, which indicates a high neutrophil count or a low lymphocyte count before treatment, represents an imbalance in the inflammatory pathway and immune response against tumors. Thus, tumor recurrence and metastasis may occur more frequently in patients with a high NLR (24). The cause of decreased lymphocyte count before treatment has not been completely elucidated, and various causes include $\mathrm{T}$ lymphocyte apoptosis via the Fas/FasL pathway and the 
Table 2 Liner regression analysis to identify variables associated with ALC and NLR

\begin{tabular}{lrcc}
\hline Variables & B & $95 \% \mathrm{Cl}$ & P value \\
\hline ALC & & & \\
Spleen V5 & -2.307 & -3.931 to -0.682 & 0.006 \\
Spleen V10 & -2.672 & -4.612 to -0.731 & 0.008 \\
Spleen V20 & -2.343 & -5.847 to 1.161 & 0.187 \\
Spleen V30 & -3.192 & -8.580 to 2.196 & 0.242 \\
Mean splenic dose & -6.380 & -13.542 to 0.782 & 0.080 \\
Increase ratio of NLR & & & \\
Spleen V5 & 0.022 & 0.004 to 0.041 & 0.019 \\
Spleen V10 & 0.022 & -0.00034 to 0.044 & 0.054 \\
Spleen V20 & 0.002 & -0.038 to 0.042 & 0.915 \\
Spleen V30 & -0.003 & -0.065 to 0.058 & 0.916 \\
Mean splenic dose & 0.045 & -0.036 to 0.127 & 0.274 \\
\hline
\end{tabular}

$\mathrm{B}$, region coefficient; $\mathrm{Cl}$, confidence interval; $\mathrm{V} 5$, volume receiving 5 Gy; V10, volume receiving 10 Gy; V20, volume receiving 20 Gy; V30, volume receiving $30 \mathrm{~Gy}$; ALC, absolute lymphocyte count; NLR, neutrophil/lymphocyte ratio.

Table 3 Logistic regression analysis to identify variables associated with grade 3 lymphopenia

\begin{tabular}{lccc}
\hline Variables & OR & $95 \% \mathrm{Cl}$ & P value \\
\hline Spleen V5 & 1.031 & $1.004-1.059$ & 0.024 \\
Spleen V10 & 1.040 & $1.004-1.078$ & 0.031 \\
Spleen V20 & 1.090 & $0.997-1.217$ & 0.121 \\
Spleen V30 & 1.159 & $0.935-1.436$ & 0.178 \\
Mean splenic dose & 1.109 & $0.985-1.247$ & 0.086 \\
\hline
\end{tabular}

OR, odds ratio; $\mathrm{Cl}$, confidence interval; $\mathrm{V} 5$, volume receiving 5 Gy; V10, volume receiving 10 Gy; V20, volume receiving 20 Gy; V30, volume receiving $30 \mathrm{~Gy}$.

presence of transforming growth factor $\beta(25,26)$. Patients with advanced-stage cancer or poor general condition may present with low ALC and high NLR at baseline before treatment. Therefore, ALC or NLR before and after treatment should be investigated. The present study first evaluated the significant difference in spleen dose and NLR before and after treatment. In increase ratio of NLR, it has been shown that a low dose spread to the spleen (i.e., V5) similar to decrease ALC was essential for NLR after treatment in patient with esophageal cancer.
In our study, only nine patients received RT alone, and this number was not comparable to that of patients who received CCRT. Additionally, some patients received chemotherapy at a lower dose due to renal dysfunction and poor general condition. Therefore, the effect of chemotherapy in decreasing ALC and increasing NLR has not been completely evaluated in our study. A study of non-small cell lung cancer patients showed that the occurrence of radiation-induced lymphopenia was independent of chemotherapy in patients with and without chemotherapy (27). Considering the decrease in ALC and neutrophil count after treatment, the effect of chemotherapy cannot be ignored. However, the decrease in ALC due to RT has a greater effect because the NLR increased after treatment. Because if chemotherapy has a significant effect, it can also decrease the neutrophil count after treatment. Therefore, the increase in radiation dose to the spleen, rather than chemotherapy, is the main factor associated with the decrease in ALC in CCRT patients.

Bone extraction was not completed with our treatment planning device, and the bone marrow dose could not be evaluated. The bone marrow is extremely vulnerable to damage caused by RT. In a previous study, a significant decrease in lymphocyte counts was observed on days 14 and 21 after whole-body irradiation in guinea pigs (28). This is an effect of total-body irradiation, and the distribution and dynamics of lymphocytes in the body must be considered. A previous research showed that about $40 \%$ of the labeled lymphocytes were present in the spleen at 30-60 min after injection. By contrast, the number of lymphocytes that entered into the bone was less than that of lymphocytes that entered into spleen, with a peak increase within 1-2 h (29). Neutrophils in the bone marrow are similarly affected by RT, however our study showed that NLR increase after RT and that lymphocytes in the spleen had a greater effect than neutrophils in the bone marrow by RT. The impact of bone marrow dose in decreasing ALC during esophageal cancer irradiation cannot be ruled out however, the spleen dose is the most important factor. In fact, a previous study revealed that no significant correlation was found between bone marrow dose and lymphopenia in patients with esophageal cancer (11).

The radiosensitivity of lymphocytes circulating in the blood is also high. However, their influence on the peripheral blood was not assessed in our study because the effect of RT on circulating lymphocytes has not been fully elucidated. Its impact on the heart, where the peripheral blood is concentrated and which is often included in the 
radiation field, was also unclear. Yovino et al. (30). showed that in a typical $60 \mathrm{~Gy}$ radiation treatment plan to the brain (2 Gy $\times 30$ fractions to an $8 \mathrm{~cm}$ diameter field), $98.8 \%$ of all circulating blood receives at least 0.5 Gy. However, it is difficult to consider these effects, which vary based on the circulating blood volume during RT, irradiation time, and number of portals. Additionally, in the case of CCRT, a split time of approximately 1 week to 10 days was inserted. Thus, it may be different from the dose received by the circulating blood via daily continuous irradiation.

A previous study reported that patients with low ALC during neoadjuvant chemotherapy is less likely to achieve pathological complete response (31). Moreover, treatmentrelated lymphopenia was found to be associated with poor survival in patients with various cancers including esophageal cancer $(15,32,33)$. However, our study did not reveal an association between treatment-related lymphopenia and a high NLR and survival. The finding may be attributed to heterogeneity in chemotherapy dose and treatment purpose among the target patients. That is, radical chemoradiation, preoperative chemoradiation, and palliative radiation were mixed. In particular, treatment methods such as chemotherapy at the time of recurrence were not constant. Moreover, age, general condition, host nutrition, previous history of double cancer, and different TNM stages may have a more significant influence on OS than lymphopenia due to splenic irradiation. The current study had several limitations. That is, this was a retrospective study conducted at a single center. Moreover, only a small number of patients were included, and the follow-up period was relatively short. Patients who were irradiated with a dose of $40 \mathrm{~Gy}$ had a higher number of re-conducted CT scans and re-created treatment plans. However, the location of the spleen changed primarily due to gastric contents. We contoured the spleen in CT which were first taken and second which taken at 40 Gy by fusion imaging, the positions could not be perfectly matched. Therefore, the spleen dose might be erroneous. The timing of obtaining laboratory results was heterogeneous, and some data were not collected immediately after treatment. Some patients had laboratory data obtained $>1$ week after at the end of treatment. Thus, the last laboratory data during treatment were used. These limitations should be addressed in future studies conducted multiple centers across several countries.

\section{Conclusions}

The present study first showed the correlation between the irradiated volume of the spleen and ALC and NLR in patients with esophageal cancer. Moreover, the spleen should be treated as a high-risk organ as in other organs such as the lungs and heart. In RT, the spleen dose must be significantly reduced. Our results may be useful in estimating spleen dose and lymphopenia and spleen dose constraints in future prospective studies.

\section{Acknowledgments}

The authors would like to thank Enago (https://www.enago.jp) for the English language review.

Funding: None.

\section{Footnote}

Reporting Checklist: The authors have completed the STROBE reporting checklist. Available at https://dx.doi. org/10.21037/tcr-21-1765

Data Sharing Statement: Available at https://dx.doi. org/10.21037/tcr-21-1765

Peer Review File: Available at https://dx.doi.org/10.21037/ tcr-21-1765

Conflicts of Interest: All authors have completed the ICMJE uniform disclosure form (available at https://dx.doi. org/10.21037/tcr-21-1765). The authors have no conflicts of interest to declare.

Ethical Statement: The authors are accountable for all aspects of the work in ensuring that questions related to the accuracy or integrity of any part of the work are appropriately investigated and resolved. The study was conducted in accordance with the Declaration of Helsinki (as revised in 2013). This study was approved by our institutional review board of Nihon University Itabashi Hospital (Ref No. RK-180213-9). All patients provided consent for their data to be used and additional consent for this retrospective analysis was waived.

Open Access Statement: This is an Open Access article distributed in accordance with the Creative Commons Attribution-NonCommercial-NoDerivs 4.0 International License (CC BY-NC-ND 4.0), which permits the noncommercial replication and distribution of the article with the strict proviso that no changes or edits are made and the 
original work is properly cited (including links to both the formal publication through the relevant DOI and the license). See: https://creativecommons.org/licenses/by-nc-nd/4.0/.

\section{References}

1. van Rossum PSN, Mohammad NH, Vleggaar FP, et al. Treatment for unresectable or metastatic oesophageal cancer: current evidence and trends. Nat Rev Gastroenterol Hepatol 2018;15:235-49.

2. Hironaka S, Ohtsu A, Boku N, et al. Nonrandomized comparison between definitive chemoradiotherapy and radical surgery in patients with $\mathrm{T}(2-3) \mathrm{N}($ any) $\mathrm{M}(0)$ squamous cell carcinoma of the esophagus. Int $\mathrm{J}$ Radiat Oncol Biol Phys 2003;57:425-33.

3. Ishikura S, Nihei K, Ohtsu A, et al. Long-term toxicity after definitive chemoradiotherapy for squamous cell carcinoma of the thoracic esophagus. J Clin Oncol 2003;21:2697-702.

4. Mebius RE, Kraal G. Structure and function of the spleen. Nat Rev Immunol 2005;5:606-16.

5. Showalter A, Limaye A, Oyer JL, et al. Cytokines in immunogenic cell death: Applications for cancer immunotherapy. Cytokine 2017;97:123-32.

6. Clark EJ, Connor S, Taylor MA, et al. Preoperative lymphocyte count as a prognostic factor in resected pancreatic ductal adenocarcinoma. HPB (Oxford) 2007;9:456-60.

7. Schmidt H, Bastholt L, Geertsen P, et al. Elevated neutrophil and monocyte counts in peripheral blood are associated with poor survival in patients with metastatic melanoma: a prognostic model. Br J Cancer 2005;93:273-8.

8. Yutong $\mathrm{H}$, Xiaoli X, Shumei L, et al. Increased Neutrophil-Lymphocyte Ratio Is a Poor Prognostic Factor in Patients with Esophageal Cancer in a High Incidence Area in China. Arch Med Res 2015;46:557-63.

9. Liu J, Zhao Q, Deng W, et al. Radiation-related lymphopenia is associated with spleen irradiation dose during radiotherapy in patients with hepatocellular carcinoma. Radiat Oncol 2017;12:90.

10. Wild AT, Ye X, Ellsworth SG, et al. The Association Between Chemoradiation-related Lymphopenia and Clinical Outcomes in Patients With Locally Advanced Pancreatic Adenocarcinoma. Am J Clin Oncol 2015;38:259-65.

11. Saito T, Toya R, Yoshida N, et al. Spleen DoseVolume Parameters as a Predictor of Treatment-related
Lymphopenia During Definitive Chemoradiotherapy for Esophageal Cancer. In Vivo 2018;32:1519-25.

12. Talsma K, van Hagen $\mathrm{P}$, Grotenhuis BA, et al. Comparison of the 6th and 7th Editions of the UICC-AJCC TNM Classification for Esophageal Cancer. Ann Surg Oncol 2012;19:2142-8.

13. Varga I, Babala J, Kachlik D. Anatomic variations of the spleen: current state of terminology, classification, and embryological background. Surg Radiol Anat 2018;40:21-9.

14. Cesta MF. Normal structure, function, and histology of the spleen. Toxicol Pathol 2006;34:455-65.

15. Zhao Q, Xu X, Yue J, et al. Minimum absolute lymphocyte counts during radiation are associated with a worse prognosis in patients with unresectable hepatocellular carcinoma. Therap Adv Gastroenterol 2017;10:231-41.

16. Ban S, Konomi C, Iwakawa M, et al. Radiosensitivity of peripheral blood lymphocytes obtained from patients with cancers of the breast, head and neck or cervix as determined with a micronucleus assay. J Radiat Res 2004;45:535-41.

17. Scott D, Barber JB, Levine EL, et al. Radiation-induced micronucleus induction in lymphocytes identifies a high frequency of radiosensitive cases among breast cancer patients: a test for predisposition? Br J Cancer 1998;77:614-20.

18. Nakamura N, Kusunoki Y, Akiyama M. Radiosensitivity of CD4 or CD8 positive human T-lymphocytes by an in vitro colony formation assay. Radiat Res 1990;123:224-7.

19. Venkatesulu BP, Mallick S, Lin SH, et al. A systematic review of the influence of radiation-induced lymphopenia on survival outcomes in solid tumors. Crit Rev Oncol Hematol 2018;123:42-51.

20. Lee YY, Choi CH, Kim HJ, et al. Pretreatment neutrophil:lymphocyte ratio as a prognostic factor in cervical carcinoma. Anticancer Res 2012;32:1555-61.

21. Käsmann L, Bolm L, Schild SE, et al. Neutrophil-toLymphocyte Ratio Predicts Outcome in Limited Disease Small-cell Lung Cancer. Lung 2017;195:217-24.

22. Mantovani A, Allavena P, Sica A, et al. Cancer-related inflammation. Nature 2008;454:436-44.

23. Sgambato A, Cittadini A. Inflammation and cancer: a multifaceted link. Eur Rev Med Pharmacol Sci 2010;14:263-8.

24. Li X, Han Z, Cheng Z, et al. Preoperative neutrophil-tolymphocyte ratio is a predictor of recurrence following thermal ablation for recurrent hepatocellular carcinoma: a retrospective analysis. PLoS One 2014;9:e110546. 
25. Kim R, Emi M, Tanabe K, et al. The role of Fas ligand and transforming growth factor beta in tumor progression: molecular mechanisms of immune privilege via Fasmediated apoptosis and potential targets for cancer therapy. Cancer 2004;100:2281-91.

26. Zhu J, Petit PF, Van den Eynde BJ. Apoptosis of tumorinfiltrating T lymphocytes: a new immune checkpoint mechanism. Cancer Immunol Immunother 2019;68:835-47.

27. Tang C, Liao Z, Gomez D, et al. Lymphopenia association with gross tumor volume and lung V5 and its effects on non-small cell lung cancer patient outcomes. Int J Radiat Oncol Biol Phys 2014;89:1084-91.

28. Akeem S, Lukman O, Eltahir K, et al. Bone Marrow and Peripheral Blood Cells Toxicity of a Single 2.0 Gy Cobalt60 Ionizing Radiation: An Animal Model. Ethiop J Health Sci 2019;29:195-202.

29. Smith ME, Ford WL. The recirculating lymphocyte pool of the rat: a systematic description of the migratory behaviour of recirculating lymphocytes. Immunology 1983;49:83-94.

Cite this article as: Sakaguchi M, Maebayashi T, Aizawa T, Ishibashi N, Okada M. Association between unintentional splenic radiation and lymphopenia and high neutrophil/ lymphocyte ratio after radiotherapy in patients with esophageal cancer. Transl Cancer Res 2021;10(12):5076-5084. doi: 10.21037/ tcr-21-1765
30. Yovino S, Kleinberg L, Grossman SA, et al. The etiology of treatment-related lymphopenia in patients with malignant gliomas: modeling radiation dose to circulating lymphocytes explains clinical observations and suggests methods of modifying the impact of radiation on immune cells. Cancer Invest 2013;31:140-4.

31. van Rossum PSN, Deng W, Routman DM, et al. Prediction of Severe Lymphopenia During Chemoradiation Therapy for Esophageal Cancer: Development and Validation of a Pretreatment Nomogram. Pract Radiat Oncol 2020;10:e16-26.

32. Shiraishi Y, Fang P, Xu C, et al. Severe lymphopenia during neoadjuvant chemoradiation for esophageal cancer: A propensity matched analysis of the relative risk of proton versus photon-based radiation therapy. Radiother Oncol 2018;128:154-60.

33. Saroha S, Uzzo RG, Plimack ER, et al. Lymphopenia is an independent predictor of inferior outcome in clear cell renal carcinoma. J Urol 2013;189:454-61. 